\title{
Self and Well-being
}

Since long the study of self has been of prime importance for the pursuit of happiness and well-being. Thinkers have shown concern for understanding and identifying the best way to achieve these states in people's lives. In recent years the quest for well-being has become a point of intersection for researchers from many disciplines. There is consensus that we are far from the desired level of well-being required to reach an experience of optimal self and therefore effort has to be made to maximize it. Much of the research on well-being, however, has been framed in a peculiar model of selfhood which is independent, separate, and bounded. This essentially Euro-American model is expressed in most of the currently popular self-related constructs (e.g. self-efficacy, internal control, self-enhancement, self-verification, and self-esteem). These concepts and demonstrations of their functional value in life serve to establish the egoistic self as a normative category.

The liberal individualism underlying the notion of an independent self promotes explicit celebration of a kind of personhood which evolves in the direction of becoming more and more individualized. To such a notion of self happiness and well-being predominantly imply hedonic qualities such as joy, excitement, possessions, and achievements. This pattern goes well with the spirit of utilitarianism. As a result the effort is centered on enhancing well-being by increasing possessions and exercising greater and greater control over the environment. One significant consequence of this view is the emergence of consumerism as a central feature of our life.

Today commodities and their buying and selling are becoming main activities in peoples' lives. Supported by strong media we are changing our life styles, ideals, and above all our own very notions of being and well-being. The ideals of body, beauty and behavior are being redefined by media. By supplying effective role models an impressive range of products is sold to people as technologies of self. In this scenario the cosmetic industry and cosmetic surgery are becoming thriving enterprises. Mass media presentation brings legitimacy to goods and they are believed to be commonplace, desirable, and within reach. The media defines good life in terms of material growth and prosperity. Since our judgments of happiness are relative and involve social comparison one is cursed to remain in a state of persistent deprivation and therefore unhappiness.

The effects of consumer culture on our lives are pervasive. For example, market is providing the vocabulary for relationships and emotions, and shopping is becoming a marker of identity. We often define ourselves in terms of what we buy. In cities our presence and participation in the shopping malls are regulating our emotions and expressions of identity. All this is making selfishness and materialism as prime life goals. People are guided by the desire of moneymaking and judge their own and others' well-being on what they possess, how they appear, and what they can buy. The materialistic orientation externalizes self-control and the objects in the world outside become critical to well-being. In such a situation while autonomy often gets curtailed the person continues to think that choice and control has increased.

The pursuit of happiness following the consumerist model, however, has many limitations. At individual level experiencing happiness is constrained by the factors such as adaptation to pleasure as well as genetically determined set point. As a result it is always short lived. Also this model does not recognize the fact that life involves positive as well as negative experiences. It has pleasure and pain both. The two make each other meaningful and emphasizing pleasure alone is only a consumerist-capitalist story and therefore a distortion that suits to the egoistic self (Ahamkara). One should not forget that suffering holds a deep transformative potential. Studies have shown that enduring pain and suffering provide meaning to life and make people resilient.

It should be remembered that happiness or lack of it is a subjective experience, at least beyond absolute deprivation conditions. It has been reported that the relationship between life satisfaction and economic growth is not linear and after certain level there is no corresponding increase in life satisfaction with increase in economic status. Happiness appears to be a function of relative income or social position rather than absolute purchasing power. While people in many developed countries are richer, they are not by same degree happier.

Also, under the impact of consumer culture everyday life experiences are becoming full of instability and inconsistency. Many people are experiencing a sense of anomie and powerlessness. Concomitant to this many changes in the family life are ensuing. There is shift from a social to instrumental function of family. Sense of obligation and commitment to family life is loosing its premium value. The trend of increase in divorce rate, domestic violence, number of non-family household, single parent families, and unmarried cohabitation is challenging the meaning and shape of the institutions of family and marriage. While 
openness of expression and dialogue, reduction in vertical hierarchies and lessening of suppression and shifts in easing power in human relationships indicate positive changes, a balance between family demands and personal fulfillment is becoming increasingly difficult. Also, the relationship between family and community too is changing. In particular migration of population from villages and small cities to the urban centers and metros is leading to decline in the traditional sources of support and decrease in spontaneity, sense of 'neighborliness', and emotional resources. In such a scenario people are experiencing loss of control over their lives.

On a closer scrutiny we find that the individualistic view of self-hood and well-being has several important limitations. A deeper analysis of happiness shows that personal happiness is inseparable from others' happiness. In an important sense happiness and well-being may be treated as ongoing processes of expansion leading toward communion. Hence we need a responsible self, one that engages with society in a dialectical relationship. This requires cultivation of the notion of an inclusive self.

Interestingly some models are available in many nonwestern as well as in western societies which offer insights about such an alternative. For example the inclusive multilayered notion of self in Indian culture (Panch Kosas) covers the physical, vital, social, mental and blissful levels of existence and situates the discourse of self in an increasingly inclusive configuration of reality. It considers selfhood as a structure in which one is embedded in the 'other', rather than in opposition to the other (e.g. person/group/community). This is so because the goal is interdependence leading to reduction of the gulf between self and not self and through that reaching increasingly complex levels of individuation but not an excluvist individualism. There exists dialectical relationship between self and other with a potential of a balance of opposite forces instead of contradiction. Also, there is clear recognition of constantly changing nature of the world and observing a holistic and encompassing perspective.

The Indian view holds that the physical reality is fluid, temporary, and of transitory nature. It is in a constant flux. Submitting to this kind of reality is constraining and oppressive as it is contingent on immediate and changing circumstances. It reflects a momentary phase within a larger cosmic existence. In this sense there is no possibility of possession and accumulation of happiness. The peace and calmness that one needs may come from involving in action in a disengaged fashion (Anasakt). It is only through quieting the ego that the necessary self-transformation can take place. Only then it will be possible to experience the bliss which is intrinsically present in each and every being. This view emphasizes self-realization or liberation which is independent from conditionings and contingencies rather than being constantly subjected to them while living in an illusion of independence and control. Therefore, it is only by moving to a non-egoic state of mind in which we are not the slaves of egocentric tendencies, the real freedom and happiness can be attained.

Being narcissistic the individualistic self fails to take note of the fact that sociality is a primary need of individuals and human development is intrinsically dialogical. It is becoming increasingly clear that development across all the domains including survival, communication, language, emotions, morality, and knowledge about the world and self is intrinsically dialogical. An independent self ignores the broader societal and ecological context within which we are embedded, live and grow. Therefore such selves would not be viable in the long run and pose serious threats to initiatives for sustainable development.

Our existence demands transcending the boundaries of body and ego and moving toward relational existence which encompasses family, community, and environment. While individualistic model of self relates to social welfare to the extent it goes well with personal gratification, in the expanded view of self the notion of happiness and wellbeing are realized in one's relationship with the surrounding realities, both social and non-social. One must not forget that sensory pleasure does not exhaust the sources of happiness. One may have pleasure in art, relationships, pro social acts, and spiritual pursuit. Here lies the need to conceptualize responsible participation in the life processes within the framework of dharma or harmonious order. We need to address simultaneously the needs of agency as well as communion and create supportive family environment, strengthen neighborhood, prioritize incentives, foster social participation and relate to broader community. Being well is the key to well-being.

Today, relating to the larger surrounding society has become a dominant concern for psychology. A shift from scholarly and scientific to professional and practical concern is visible in the journey of the discipline. But the question that we need to address is this: should we sit as a silent observer and accept the socio-political and economic structures uncritically and become collaborators in the dehumanizing process? We do share social responsibility and it is imperative that we must make conscious moral choices rather than surrendering to the ideology of those who are in power. Let us move forward to humanize the discipline and work towards creating a world where there is peace and happiness and people are able to make sense of life and world and have faith, hope, and courage.

Girishwar Misra

Editor 